\title{
MARKETING B2B: MAPEAMENTO DOS TRABALHOS ACADÊMICOS NO BRASIL DE 1998 A 2007
}

\author{
B2B MARKETING: MAPPING ACADEMIC PAPERS \\ IN BRAZIL FROM 1998 TO 2007
}

Tales Sarmento Lacerda ${ }^{1}$ e Beatriz Quaresma Mendonça ${ }^{2}$

\section{Resumo}

O Marketing, ao mesmo tempo em que é rico por receber contribuições de áreas diversas, como Economia, Sociologia e Psicologia, dentre outras, ainda busca consolidar uma teoria própria. Nesse sentido, a classificação de trabalhos acadêmicos apresenta-se como importante para o mapeamento dos rumos em que o Marketing vem sendo trabalhado ao longo dos últimos anos no Brasil, assim como para o levantamento de tendências da área. Entender as características da compra organizacional é essencial para a criação de programas eficazes de marketing que atinjam os compradores deste mercado. Isso porque tais compradores não se comportam como os de mercado de consumo, apesar do objetivo na realização da compra ser o mesmo em ambos os casos - satisfazer uma necessidade. Os objetivos e as políticas singulares de uma organização estabelecem restrições especiais na tomada de decisão do comprador organizacional. (KERIN et al., 2007). Este estudo buscou identificar os artigos da área de Marketing Business-to-Business (B2B) publicados nos EnANPADs, RAE, RAC, READ e RAUSP no período de 1998 a 2007, verificando, apesar da pequena quantidade de trabalhos realizados, o número tem aumentado e, seguindo a tendência da literatura, as pesquisas estão se concentrando na área de marketing de relacionamento entre empresas.

Palavras-chave: Marketing business-to-business; Mapeamento de trabalhos; Teoria de marketing; Estudo de marketing no Brasil.

\footnotetext{
1 Mestre em Administração - CEPEAD/UFMG. Professor na Pós-Graduação Lato Sensu - FUMEC, IEC-Puc Minas e CEPEAD/UFMG. E-Mail: tales@task.com.br

2 Especialista em Gestão de Negócios - CEPEAD/UFMG. E-mail: beatriz@takono.com.br
} 


\begin{abstract}
The Marketing discipline is influenced by several areas like Economics, Sociology and Psychology. However, it's still looking for an own theory. The scientific classification of papers becomes an important tool to find the way Marketing has been studied on last years in Brazil, as well as to find trends in this area. Understanding the organizational buying characteristics is mandatory in order to create efficient marketing programs that meet industry's buyers. This occurs because those buyers don't have the same behavior as individual consumers, despite the goal of a buying operation be the same - satisfaction of a need. The goals and politics of a particular organization offer restrictions on buying decision of organizational buyer. (KERIN et al., 2007). So, this paper tried to identify Business-to-Business (B2B) Marketing work published on EnANPADs, RAE, RAC, READ e RAUSP between 1998 and 2007. Results point that, despite the small amount of papers, the number is increasing and, following the literature trend, research are focusing on organizational relationship marketing.

Keywords: Marketing business-to-business; Work mapping; Marketing theory; Marketing study on Brazil.
\end{abstract}

\title{
Introdução
}

O marketing como corpo de conhecimento e disciplina acadêmica deve muito ao que Bartels (1976) chama de período de reconceitualização, quando o conceito de marketing e o paradigma do mix de marketing (4 Ps), introduzido nas décadas de 1950 e 1960, definiram a natureza e o conteúdo de seu gerenciamento. Contudo, para Malley e Tynan (2005), a abordagem do gerenciamento do mix de marketing concentra-se na venda de produtos a consumidores, sendo que grande parte do marketing ocorre em outras situações que não essa; por exemplo, quando o comprador é uma empresa e não um consumidor individual.

Segundo Webster e Wind (1972), a ênfase da literatura sobre os comportamentos de compra sempre esteve nos consumidores finais, e muito pouco foi pesquisado e estudado sobre o comportamento de compradores organizacionais, sendo ainda que as pesquisas encontradas e discussões teóricas sobre comportamento do consumidor final têm pouca relevância no mercado organizacional. Nesse sentido, a classificação de trabalhos acadêmicos apresenta-se como importante para o mapeamento dos rumos em que o estudo das relações mercadológicas entre empresas - Marketing B2B, também denominado Marketing Empresarial ou Marketing Industrial - vem sendo trabalhado ao longo dos últimos anos no Brasil, assim como para o levantamento de tendências da área.

Esta pesquisa buscou localizar os trabalhos de marketing B2B nas principais revistas de Administração no Brasil, com conceito "A" pelo critério do Qualis da CAPES (Coordenação de Aperfeiçoamento de Pessoal de Ensino Superior): Revista de Administração de Empresas (RAE), Revista de Administração Contemporânea (RAC), Revista de Administração da USP (RAUSP) e Revista Eletrônica de Administração (READ), e dos anais dos encontros anuais da Associação Nacional dos Programas de Pós-Graduação em Administração (EnANPADs), uma vez que é o principal evento acadêmico de Administração no Brasil (VIEIRA, 1998), com edições regulares ao longo dos últimos 31 anos. Tendo em vista o caráter representativo do ENANPAD, e considerando que entre as suas diversas áreas temáticas encontra-se a de Marketing, o presente artigo assume o pressuposto, inicialmente adotado por Vieira (1998), de que os trabalhos publicados nos Anais do ENANPAD refletem a produção acadêmica, bem como o esforço de pesquisa em Marketing dos principais centros de pós-graduação do país. Assim, entende-se que a publicação

Rev. Adm. UFSM, Santa Maria, v. 3, n. 2, p. 219-229, mai./ago. 2010 
científica resultante desse encontro, na área de Marketing e das citadas revistas, caracteriza-se como uma referência na construção e desenvolvimento críticos do Marketing no Brasil.

\section{Buscando uma Teoria de Marketing}

Enquanto as primeiras ideias sobre Marketing podem ser datadas dos anos 1880, a maioria dos estudiosos concorda que o Marketing realmente adquiriu uma forma mais consistente em meados da década de 1950, início dos anos 1960, com contribuições seminais de autores como Peter Drucker, Joel Dean, Theodore Levitt e Eugene McCarthy (BAKER, 2000b).

Baker (2000a) mostra que, apesar da verdadeira essência do marketing ser o estabelecimento de relações de troca que levem à mútua satisfação, pode-se encontrar mais de uma centena de definições do que vem a ser o marketing, que variam em relação ao seu objeto de estudo, natureza do relacionamento, resultados, aplicações e à dicotomia filosofia x função. Para o autor, as mudanças ocorridas ao longo do tempo nesses cinco temas indicam a significativa evolução do conceito de marketing desde sua primeira definição. Entretanto, ainda que se deva exigir maior rigor na formulação de definições no futuro, unificar a definição desencorajaria, provavelmente, o desenvolvimento do assunto. Baker (2000a) recomenda ainda que se busque estabelecer ao menos uma distinção entre o marketing como uma filosofia de negócios, que guia o desenvolvimento e direciona uma organização, e aquele visto como uma função de negócios, aumentando a eficiência e eficácia na execução da missão da organização.

A partir da década de 1960, com a questão dos efeitos nocivos da talidomida, o marketing começa a se preocupar com a segurança dos produtos. Na década de 1970, aumenta a publicação de artigos sobre o impacto do marketing na sociedade, e se começa a criticar as práticas de marketing que se baseiam somente na exploração dos recursos naturais e não se preocupam com o bem-estar da sociedade. Ainda segundo Sheth, Gardner e Garrett (1988), a década de 1980 trouxe à tona questões como o estudo de grupos de consumidores (como idosos, minorias étnicas, crianças) e as relações saudáveis no marketing, bem como a preocupação macroambiental do marketing (Macromarketing), envolvendo discussões sobre intervenções legais, legislação de propaganda, restrições de distribuição, controle e política de preço, dentre outros.

\section{Problemática e relevância - publicações de marketing}

Assim como acontece com a própria Administração, que ainda se questiona ser ou não uma ciência, o marketing também levanta o debate sobre ser uma ciência em si mesmo ou apenas uma área de aplicação das descobertas das ciências (basicamente das ciências comportamentais). (SAREN, 2000). Para BARTELS (1951), para que o marketing seja reconhecido como ciência é necessário o estabelecimento de leis gerais ou princípios mais abrangentes, bem como que as previsões decorrentes do desenvolvimento dessas leis seja relevante para a sociedade, e não apenas para as instituições.

Cabe ressaltar que não se pretende aqui esgotar o assunto ou se chegar a uma avaliação de forma definitiva, apesar de haver, de certa forma, um consenso em relação aos elementos necessários para a construção teórica (BOTELHO; MACERA, 2001). Para as autoras, que analisaram teses e dissertações publicadas na FGV-EAESP, de 1974 a 1999, o debate está em como avaliar teorias, pois "a ausência de uma metateoria em marketing é ao mesmo tempo causa e consequência das dificuldades pelas quais atravessa a construção teórica da disciplina" (BOTELHO; MACERA, 2001, p. 2). 
No Brasil, o trabalho pioneiro de Machado-da-Silva, Cunha e Amboni (1990) despertou a atenção da academia de Administração para a necessidade de voltar seu olhar para sua produção, avaliar sua 'evolução'. Tal estudo avaliou os artigos da área de Organizações, diagnosticando uma fragilidade teórico-metodológica e um predomínio de uma abordagem funcionalista. Foi um primeiro passo para que várias áreas iniciassem balanços críticos de sua produção, inclusive a de marketing.

Por sua vez, na área de Marketing, um autor que realizou um amplo balanço crítico foi Vieira (1998, 1999, 2000). O trabalho de 1998, por meio de uma avaliação dos artigos publicados nos Anais do ENANPAD durante a década de 1990, diagnosticou uma concentração dos temas de pesquisa em Comportamento do Consumidor, Estratégia de Mercado, Marketing de Serviços e Sistema de Informação de Pesquisa em Marketing, com uma concentração das publicações acadêmicas na área na UFRGS, USP e UFRJ. Um estudo de Perin et al. (2000), do mesmo objeto (Anais do ENANPAD da década de 1990), afirma que as análises de pesquisas empíricas do tipo survey apresentam qualidade no mínimo questionável.

No estudo de Vieira (2000), 272 artigos (trabalhos resultados de pesquisas teóricas, empíricas, ensaios etc.) de marketing foram publicados no Brasil de 1990 a 1999 - considerados os Anais do ENANPAD e as revistas de Administração (RA) e de Administração de Empresas (RAE). As principais temáticas estudadas foram comportamento do consumidor (18,8\%), serviços $(10,3 \%)$ e estratégias de mercado $(9,9 \%)$. Houve, portanto, um nítido direcionamento dos estudos e pesquisas no sentido de se conhecer melhor o mercado consumidor brasileiro. Vários estudos foram publicados sobre aspectos multidisciplinares e pluralísticos de marketing $(10,7 \%)$. De certa forma, tal volume de trabalhos implica a existência de um diálogo constante do marketing com outras áreas de conhecimento e, de algum modo, revela certa dependência do conhecimento oriundo de outras áreas para a construção e consolidação de um estatuto teórico que lhe seja particular.

Um estudo que utilizou as escolas definidas por Sheth, Gardner e Garrett (1988), para classificar os trabalhos disponibilizados nas bases de dados do Proquest, Emerald, Bibliotecas Virtuais das Universidades de Harvard, Columbia e MIT (Messachusetts Insitute of Technology Estados Unidos) e livrarias virtuais, foi o de Miranda e Arruda (2002). Por meio de uma metodologia qualitativa, na qual a desk research foi a técnica utilizada, foi identificado o foco dominante das doze escolas emergidas no decorrer do século XX, realizado um levantamento teórico documental de fontes secundárias dos acessos descritos. Como resultado, diagnosticouse que os estudos desenvolvidos na década de 1990 focalizaram a estrutura dos relacionamentos, com concentração em satisfação e retenção de clientes, emergindo, neste novo século, uma maior ênfase nos relacionamentos de interatividade das transações comerciais entre vendedores e consumidores, estes últimos vistos como o ponto central do marketing, criando novos conceitos, como cybermarketing e marketing experiencial.

Outro artigo publicado nos Anais do ENANPAD de 2002, de Vieira et al. (2002), realizou uma análise dos os trabalhos de marketing publicados nos anais do referente congresso entre os anos de 1997 e 2001, levantando evidências de que as publicações desta área estão fortemente orientadas pelas ciências empírico-analíticas, fato que traz à tona uma lacuna no discurso crítico das teorias de marketing e a necessidade de adoção de uma perspectiva emancipatória para que se possa capturar a essência de um projeto filosófico diferente. Outra análise dos autores refere-se à percepção, levando em conta o Brasil como cenário, de que a grande maioria dos estudos possui uma perspectiva superficial, na medida em que tentam sem sucesso ser abrangentes, não sendo nem genéricos nem específicos. Além disso, na área de 
marketing, a produção brasileira reproduz as oscilações e os modismos de inspiração quase exclusivamente norte-americanos.

Mais um artigo de Vieira (2003), publicado na Revista de Administração de Empresas, apresenta uma 'fotografia' da produção brasileira de marketing em termos de temática, número de autores e citações por artigo e número de citações de periódicos nacionais e internacionais, constatando que os pesquisadores nacionais não têm utilizado como referência a publicação científica local. Faria et al. (2006) pesquisaram a área de marketing dos EnANPADs de 2000 a 2005, verificando uma tendência de aumento dos trabalhos na área de comportamento do consumidor, além de notar muito presente a questão da multidisciplinaridade, que, como abordada por Hunt (2002), ao mesmo tempo em que propicia um enriquecimento da produção acadêmica na área, de certa forma distancia a construção de uma teoria geral do marketing.

Do pesquisado em relação às publicações em marketing de modo geral, mais notadamente no Brasil, existe todo um questionamento, um olhar voltado para a produção acadêmica de modo mais atento, a partir da década de 1990, apesar de serem verificadas poucas mudanças no sentido de melhorias em termos de quantidade, diversidade e qualidade, da produção científica da área.

\section{Marketing business-to-business e compras organizacionais}

Entender as características da compra organizacional é essencial para a criação de programas eficazes de marketing que atinjam os compradores deste mercado. Isso porque tais compradores não se comportam como os de mercado de consumo, apesar do objetivo na realização da compra ser o mesmo em ambos os casos - satisfazer uma necessidade. Os objetivos e as políticas singulares de uma organização estabelecem restrições especiais na tomada de decisão do comprador organizacional. (KERIN et al., 2007).

As compras organizacionais envolvem mais pessoas no processo de decisão com complexas interações entre essas, suas metas pessoais e os objetivos da empresa. (WEBSTER; WIND, 1970). Segundo Kerin et al. (2007), a compra organizacional tem maior probabilidade de envolver negociações demoradas em relação a cronograma de entrega, preço, especificações técnicas, garantias e políticas de indenização. Para Hutt e Speh (2002), algumas vezes os produtos são iguais, mas são necessárias abordagens de marketing fundamentalmente diferentes para alcançar o comprador organizacional.

Empresas que vendem produtos ou serviços de consumo tentam atingir milhares ou milhões de indivíduos. Em comparação, as empresas que vendem para organizações são frequentemente restritas a muito menos compradores potenciais. Assim, as empresas realizam compras que evolvem mais dinheiro e maior quantidade de produtos do que as feitas por consumidores. Para Kotler (2000), isso resulta no fato de os fornecedores customizarem suas ofertas com intuito de atender às necessidades de cada empresa. Às vezes, os compradores exigem que os vendedores revejam não apenas suas práticas, como também seu desempenho, e os relacionamentos entre clientes e fornecedores têm mudado de desagradáveis e adversários para próximos e amigáveis.

Kotler (2000) cita como diferencial as obrigações de seguir as políticas, normas e exigências da organização e a existência de muitos trâmites - como cotações, propostas e contratos - que não são vistos com frequência em compras realizadas por consumidores.

Assim, a realidade do marketing per se é que fornecedores enfrentam mercados concentrados nos quais clientes individuais podem ter importância crucial. Esses clientes não são 
passivos, mas pesquisam e interagem ativamente com fornecedores selecionados e reclamam produtos customizados. (TURNBULL; LEEK, 2005).

Existe uma ligação direta entre a demanda por produto industrial e a demanda por produtos de consumo: a primeira deriva-se da segunda. Para Hutt e Speh (2002), em razão do fato de a demanda ser derivada, o profissional de marketing B2B deve monitorar cuidadosamente os padrões de demanda e as mudanças nas preferências dos consumidores no mercado de consumo, normalmente em bases mundiais, e não somente monitorar, mas também desenvolver programas que atinjam diretamente o consumidor final.

Algumas outras características diferenciam a estratégia do marketing organizacional. Para Hutt e Speh (2002), o profissional de marketing B2B deve enfatizar a venda pessoal em vez de anunciar (TV, jornal) para atingir compradores em potencial. Para os autores, apenas uma parte do orçamento promocional deverá ser investida em propaganda (feita mais comumente através de jornais mercantis ou mala direta). Essa propaganda, no entanto, normalmente estabelece a base para uma visita de vendas bem-sucedida.

Segundo Kerin et al. (2007), para compras rotineiras, com pequeno volume monetário, um único comprador ou gerente de compra frequentemente toma a decisão de compra sozinho. Contudo, em muitos casos, diversas pessoas na organização participam do processo de compra. Os indivíduos deste grupo, denominado centro de compras, compartilham metas, riscos e conhecimentos importantes para uma decisão de compra. Os membros do centro de compras são motivados por uma complexa interação de metas e objetivos individuais e organizacionais. O profissional de marketing empresarial deve estar mais preocupado em descobrir quem são as pessoas influentes nos diferentes tipos de organizações, em cada estágio do processo decisório, e seu grau relativo de influência. (TURNBULL; LEEK, 2005).

As relações em que não há dependência de um único fornecedor nem comprometimento entre as partes e que objetiva a satisfação de interesses de curto prazo, precisam de ações do Marketing Transacional. (VLOSKY; WILSON, 1997). As ideias tradicionais sobre as compras organizacionais, baseadas na otimização de uma única transação, enfatizam trocas de soma-zero, em que o ganho de um dos lados leva à perda do outro, tornando as empresas mais adversárias do que colaboradoras.

No entanto, àquelas relações em que o comportamento de compra focaliza, inegavelmente, uma construção de um relacionamento de longo prazo, por meio de alto grau de confiança e compromisso, devem-se aplicar as ações do marketing de relacionamento. O conceito de marketing de relacionamento, em virtude de sua complexidade e de seu caráter multidimensional, é operacionalizado pela comunidade científica como conjunto de variáveis distintas, as quais indicam uma maior orientação da empresa para a manutenção de trocas com foco no longo prazo e com espírito ganha-ganha. Assim, o marketing de relacionamento concentra todas as atividades de marketing com a finalidade de estabelecer, desenvolver e manter relacionamentos bem-sucedidos com o cliente. (HUTT; SPEH, 2002).

A provável inadequação das teorias transacionais do comportamento do comprador empresarial, face às contingências do ambiente competitivo contemporâneo, opinião prevalecente entre expressivos autores e pesquisadores de business-to-business (B2B), acrescida da necessidade de examinar com maior profundidade as relações cliente-fornecedor, abre uma importante vertente de pesquisa sobre o tema marketing de relacionamento. (SIQUEIRA; TOLEDO, 2004).

Segundo Hutt e Speh (2002), a nova era de marketing B2B baseia-se em um gerenciamento efetivo de relacionamento. Para os autores, um relacionamento forte com seus clientes assegura vantagens importantes e duradouras, que os concorrentes têm dificuldade de 
entender, copiar ou desfazer. O marketing de relacionamento é, pois, uma ideia crescente na economia mundial, e se apoia na necessidade de uma mudança nas relações empresariais, em que a confrontação e o poder de barganha deem lugar à cooperação e ao desenvolvimento de um relacionamento comercial colaborativo. (HAKANSSON, 1982).

Muitas empresas estão, hoje, mudando sua ênfase do marketing de transação para o marketing de relacionamento. Os clientes de hoje são grandes e geralmente globais, por isso preferem fornecedores que possam vender e entregar uma série de produtos e serviços coordenados para muitos locais, que possam rapidamente resolver problemas e trabalhar com as equipes de clientes para melhorar seus produtos e processos. (KOTLER, 2000).

Devido à crescente importância das relações estratégicas de longo prazo tanto com clientes como com fornecedores, as empresas valorizam cada vez mais as habilidades de gerenciamento de relacionamento. Considerando que essas habilidades estão muito mais nas pessoas do que em empresas, papéis ou tarefas, o pessoal de marketing que possuir tais habilidades passará a ser considerado um ativo muito valioso para a organização. (HUTT; SPEH, 2002).

Kotler (2000) sugere que as empresas devem fazer com que seus vendedores abandonem abordagens preliminares para investigar os problemas e as necessidades do cliente potencial, demonstrar capacidades superiores do fornecedor e depois obter um compromisso de longo prazo. Essa abordagem reflete o interesse crescente de muitas empresas em desistir de perseguir uma venda imediata para desenvolver um relacionamento de longo prazo com o cliente.

De acordo com Hutt e Speh (2002), as empresas que adotam o gerenciamento da cadeia de suprimentos também solicitam ideias de seus principais fornecedores, além de envolvêlos no processo de desenvolvimento de novos produtos. Ao gerenciar os custos e a cadeia de suprimentos e utilizar a habilidade dos fornecedores no desenvolvimento de novos produtos, o departamento de compras contribuiu para o avanço do desempenho corporativo em muitas organizações.

Segundo Turnbull e Leek (2005), a percepção dos profissionais de marketing sobre a satisfação das necessidades dos clientes é alcançada dando-se maior ênfase aos processos e aos relacionamentos que ocorrem entre e dentro de organizações compradoras e vendedoras. Os profissionais de marketing devem ficar atentos às alterações nas necessidades dos clientes e devem ser sensíveis às forças competitivas que dirigem as decisões de compras dos clientes. (HUTT; SPEH, 2002).

Portanto, para contribuir no desenvolvimento de ações de marketing mais eficazes, é necessário que se entenda como é a relação de troca esperada pelo cliente, sabendo-se que uma estratégia errada pode acarretar em custos altos e poucos resultados. (HUTT; SPEH, 2002). Segundo Vlosky e Wilson (1997), programas de marketing B2B com orientação à construção de vínculos fortes e duradouros com clientes podem ser extremamente bem-sucedidos em contextos apropriados, ao passo que podem vir a ser dispendiosos e pouco efetivos em terrenos onde há ênfase em vendas individuais. Para Malley e Tynan (2005), é importante considerar como cada relacionamento deve ser gerenciado, isto é, se todos os clientes devem ser tratados do mesmo modo, que recursos devem ser investidos em cada relacionamento, e como o portfólio de relacionamentos deve ser gerenciado.

\section{Metodologia aplicada}

Este estudo caracteriza-se como descritivo, segundo Malhotra (2001), uma vez que se estabeleceu, como objetivo, identificar e classificar os artigos publicados na área de Marketing B2B nos ENANPADs e nas principais publicações brasileiras de Administração do ano de 1998 a

Rev. Adm. UFSM, Santa Maria, v. 3, n. 2, p. 219-229, mai./ago. 2010 
2007. Tais artigos são os objetos empíricos em questão neste trabalho. A classificação seguiu a divisão de áreas da ANPAD, conforme apresentado no Quadro 1.

Quadro 1 - Divisão atual de artigos de Marketing no ENANPAD

\begin{tabular}{c}
\hline \hline MKT - Marketing - Áreas Temáticas \\
\hline MKT-A Métodos de Pesquisa e Teoria em Marketing \\
MKT-B Comportamento do Consumidor \\
MKT-C Gestão e Estratégia de Marketing \\
\hline MKT-D Marketing em Contextos Específicos \\
\hline \hline
\end{tabular}

Fonte: ANPAD (http://www.anpad.org.br).

Para tanto, seguiu-se uma estratégia qualitativa, de acordo com o procedimento de pesquisa bibliográfica, analisando cada um dos artigos dos Anais do ENANPAD da área de Marketing e das revistas RAE, RAC, RAUSP e READ, do ano de 1998 até 2007, tendo como variável analisada a temática geral do estudo de cada artigo. O presente estudo baseou-se na metodologia de coleta e análise dos mais citados meta-estudos nas diversas áreas do ENANPAD, como, por exemplo, os trabalhos de Vieira (1998) e de Perin et al. (2000).

As etapas de classificação dos artigos consistiram em: leitura do artigo e categorização em uma das áreas de conhecimento de Marketing selecionadas. Cada etapa foi cumprida pelos autores deste estudo. Em caso de dúvida acerca do tema do artigo em questão, em função da apresentação, por exemplo, de características pertencentes a mais de uma área, os autores analisaram mais profundamente as referências bibliográficas, teoria e objetivos apresentados.

\section{Resultados e análises}

A tabela 1 mostra o resultado das análises feitas pelos autores, com o número de trabaIhos encontrados por referência (EnANPAD e revistas).

Tabela 1 - Relação de artigos sobre Marketing B2B publicados nos ENANPADs, RAE, RAC, RAUSP e READ de 1998 a 2007

\begin{tabular}{|c|c|c|c|c|c|}
\hline Ano / Referência & EnANPAD & RAE & RAC & RAUSP & READ \\
\hline 1998 & & & & & \\
\hline 1999 & 1 & & & & \\
\hline 2000 & 1 & & & & \\
\hline 2001 & 1 & & 1 & 1 & \\
\hline 2002 & 1 & & & 1 & \\
\hline 2003 & & & & & \\
\hline 2004 & 1 & 1 & 1 & & 1 \\
\hline 2005 & 3 & 1 & & & \\
\hline 2006 & & 1 & & & 1 \\
\hline 2007 & 3 & & & & \\
\hline Total & 11 & 3 & 2 & 2 & 2 \\
\hline
\end{tabular}

Fonte: Elaborada pelos autores. 
A análise das publicações no período (1998-2007) não permite que se estabeleçam conclusões acerca de tendências de crescimento ou declínio, mas pode-se notar uma concentração dos trabalhos nos últimos cinco anos, comparados aos cinco primeiros (13 contra 7). Mais importante, nota-se um número muito pequeno de trabalhos sobre marketing B2B com relação ao total publicado, principalmente nos EnANPADs.

A tabela 2 mostra os artigos identificados como sendo de marketing B2B, subdivididos em áreas. Não houve artigos teóricos que se enquadrassem na área MKT-A, e uma área "Marketing de Relacionamento", apesar de não existir separadamente na ANPAD, foi colocada devido à concentração de artigos que trabalham este tema.

Tabela 2 - Relação de artigos sobre Marketing B2B publicados nos ENANPADs, RAE, RAC, RAUSP e READ de 1998 a 2007, por área de conhecimento

\begin{tabular}{cccc}
\hline \hline $\begin{array}{c}\text { Ano / } \\
\text { Referência }\end{array}$ & $\begin{array}{c}\text { Marketing de } \\
\text { Relacionamento }\end{array}$ & $\begin{array}{c}\text { Comportamento do } \\
\text { Consumidor } / \\
\text { Processo de decisão } \\
\text { de Compra }\end{array}$ & $\begin{array}{c}\text { Gestão e Estratégia de } \\
\text { Marketing }\end{array}$ \\
\hline 1998 & 1 & & 2 \\
1999 & 1 & 1 & \\
2000 & 1 & 1 & 1 \\
2001 & 3 & & 3 \\
2002 & 1 & & 1 \\
2003 & 2 & & 7 \\
2004 & 2 & 2 & \\
2005 & 11 & & \\
2006 & & & \\
\hline Total & & & \\
\hline \hline
\end{tabular}

Fonte: Elaborada pelos autores.

Vale ressaltar a dificuldade encontrada para a classificação dos artigos em apenas uma das áreas de marketing selecionadas. Por exemplo, houve trabalhos que relacionaram a lealdade de clientes ao gerenciamento da cadeia de suprimentos (remetendo às áreas MKT-B - Comportamento do Consumidor e MKT-C - Gestão da distribuição, respectivamente). Em casos como esse, a definição da área em que foi classificado o trabalho deu-se em função da predominância da fundamentação teórica, referências e os objetivos, em uma análise mais detalhada.

Além disso, o Marketing B2B é classificado, na ANPAD, dentro da área MKT-D (Marketing em Contextos Específicos), mas aspectos relacionados ao Marketing B2B estão presentes nas outras áreas, como Comportamento do Comprador Organizacional (MKT-B) e Gestão e Estratégia de Marketing (MKT-C).

\section{Conclusões}

A principal conclusão é o fato de haver uma pequena quantidade de artigos publicados, mesmo levando-se em conta o EnANPAD e quatro das principais publicações da área no Brasil. Entretanto, nota-se que houve um aumento nos trabalhos relacionados ao marketing B2B quan- 
do comparados os cinco últimos anos ao período anterior (1998-2002): foram 7 de 1998 a 2002 e 13 de 2003 a 2007.

É importante ressaltar que, além da possibilidade de a área realmente estar recebendo mais atenção dos pesquisadores, até 2002 muitos trabalhos se dedicavam ao estudo do relacionamento fornecedor-cliente do ponto de vista da gestão da cadeia de suprimentos, não ficando explícita a sua relação com o marketing. O relacionamento ideal seria o que abrangesse eficiência administrativa, qualidade dos produtos e serviços, menores custos e maior produtividade (ARKADER, 1998). Com a difusão do conceito de marketing de relacionamento e a necessidade de interação entre as áreas operacional, estratégica e comercial para uma relação duradoura, passou a ser mais fácil caracterizar os trabalhos como sendo de marketing B2B.

A partir dos anos 1990, o 'Marketing de relacionamento' se tornou o tema dominante (BAKER, 2000a), como um retorno ao mais simples fundamento do Marketing: o de satisfazer mutuamente as partes em uma relação de troca. De fato, é difícil conceber, dentro da conceitualização contemporânea, um problema ou questão de marketing que não tenha em seu núcleo a ideia de construir, manter e encerrar relacionamentos, sendo que fazer bem o marketing de relacionamento é simplesmente fazer melhor o marketing tradicional (MALLEY; TYNAN, 2005).

Apesar de o marketing estar voltando, nos últimos anos, seu olhar para o produzido, tendo em vista os estudos bibliométricos e metateóricos que vêm sendo publicados, a reflexão precisa ser expandida para além das análises como as instituições de origem dos pesquisadores, o número de publicações por autor, e buscar uma maior autocrítica. Um maior conhecimento acerca da própria área, suas potencialidades, limitações, apresentar-se-ia como uma oportunidade para a evolução da produção de marketing e, quem sabe, da consolidação de uma teoria. Os primeiros passos, em uma direção comum (mas com diversidade), necessitam ser dados.

Como sugestão para futuras pesquisas, pode-se avaliar em eventos internacionais qualificados, se a mesma distribuição de trabalhos dentro das referidas escolas e no mesmo período é observada, mostrando, portanto, se esta pequena quantidade de trabalhos de marketing B2B é um fenômeno geral ou característico do Brasil.

\section{Referências}

ARKADER, R. Benefícios e problemas nas relações de fornecimento enxuto: indicações na indústria automobilística brasileira. Revista de Administração Contemporânea, v. 2, n. 1, p. 127-142, jan./abr. 1998.

BAKER, M. J. Marketing - philosophy or function? In:__. Marketing theory: a student text. New York: Thomson Learning, 2000a. p. 1-20.

The future of marketing. In: . Marketing theory: a student text. New York: Thomson Learning, 2000b. p. 293-314.
BOTELHO, D.; MACERA, A. Análise metateórica de teses e dissertações da área de marketing apresentadas na EAESP-FGV (1974-1999). In: ENANPAD, 25. 2001, Campinas. Anais eletrônicos... Campinas: ANPAD, 2001.

BARTELS, R. Can marketing be a science? Journal of Marketing, v. 15, p. 319-328, Jan. 1951.

. The history of marketing thought. 2. ed. 1976. Disponível em: <http:// www.faculty.missouristate.edu/c/ChuckHermans/ Bartels.htm >. Acesso em: 20 abr. 2008.

FARIA, P. do C. N. et al. Mapeamento, análise e classificação dos trabalhos acadêmicos de marketing nos ENANPADs de 2000 a 2005. In: EMA - ENCONTRO DE MARKETING DA ANPAD, 2., 2006, Rio de Janeiro. Anais eletrônicos... Rio de Janeiro, 2006. 
H?KANSSON, Håkan. International marketing and purchasing of industrial goods: an interaction approach. Chichester, England: John Wiley and Sons, 1982.

HUNT, Shelby D. Foundations of marketing theory: toward a general theory of marketing. Armonk, NY: ME Sharpe, 2002.

HUTT, M. D.; SPEH, T. W. B2B: gestão de marketing em mercados industriais e organizacionais. Porto Alegre: Bookman, 2002.

KERIN, Roger A. et al. Marketing. São Paulo: McGraw-Hill, 2007.

KOTLER, P. Administração de marketing: a edição do novo milênio. São Paulo: Pearson, 2000.

MACHADO-DA-SILVA, C. L., CUNHA, V. C., AMBONI, N. Organizações: o estado da arte da produção acadêmica no Brasil. In: ENANPAD, 14., 1990, Florianópolis. Anais eletrônicos... Florianópolis: ANPAD, 1990.

MALHOTRA, N. K. Pesquisa de marketing: uma orientação aplicada. Porto Alegre: Bookman, 2001.

MALLEY, L. O'; TYNAN, C. Marketing de relacionamento. In: BAKER, Michael J. Administração de marketing. Rio de Janeiro: Elsevier, 2005.

MIRANDA, C. M. C.; ARRUDA, D. M. A evolução do pensamento de marketing: uma análise do corpo doutrinário acumulado no século XX. In: ENANPAD, 26., 2002, Salvador, Anais eletrônicos... Salvador: ANPAD, 2002.

PERIN, M. G. et al. A pesquisa survey em artigos de marketing nos ENANPADs da década de 90. In: ENANPAD, 24., 2000, Florianópolis. Anais eletrônicos....Florianópolis: ANPAD, 2000.

SAREN, M. Marketing theory. In: BAKER, M. Marketing theory: a student text. New York: Thomson Learning, 2000. p. 21-42.

SHETH, J. N.; GARDNER, D. M.; GARRETT, D. E. Marketing theory: evolution and evaluation. New York: John Wiley \& Sons, 1988.

SIQUEIRA, A.C.B; TOLEDO, G.L. Comportamento do comprador industrial: uma análise comparativa entre um modelo transacional e um modelo de relacionamento. In: SEMEAD - SEMINÁRIOS DE ADMINISTRAÇÃO FEA-USP, 7., 2004, São Paulo. Anais eletrônicos... São Paulo: FEA-USP, 2004.
TURNBULL, P. W.; LEEK, S. Marketing business-tobusiness: comportamento de compra, relacionamentos e redes organizacionais. In: BAKER, Michael J. Administração de marketing. Rio de Janeiro: Elsevier, 2005. p. 103-119.

VIEIRA, F. G. D. Por quem os sinos dobram? Uma análise da publicação científica na área de marketing do ENANPAD. In: ENANPAD, 22., 1998, Foz do Iguaçu. Anais eletrônicos... Foz do Iguaçu: ANPAD, 1998.

Ações empresariais e prioridades de pesquisa em marketing: tendências no Brasil e no mundo segundo a percepção dos acadêmicos brasileiros. In: ENANPAD, 23., 1999, Foz do Iguaçu. Anais eletrônicos... Foz do Iguaçu: ANPAD, 1999.

Panorama acadêmico-científico e temáticas de estudos de marketing no Brasil. In: 24 ENANPAD, 24., 2000, Foz do Iguaçu. Anais eletrônicos... Florianópolis: ANPAD, 2000.

Narciso sem espelho: a publicação brasileira de marketing. Revista de Administração de Empresas, v. 43, n. 1, jan./fev./mar. 2003.

VIEIRA, R. S. G. et al. O conhecimento de marketing sob os olhos da teoria crítica. In: ENANPAD, 16. 2002, Salvador. Anais eletrônicos.... Salvador: ANPAD, 2002.

VLOSKY, R. P.; WILSON, E. J. Partnering and traditional relationships in business marketing: an introduction to the special issue. Journal of Business Research, v. 39, p.1-4, 1994.

WEBSTER, F. E. Jr.; WIND, Y. A general model for understanding organizational buying behavior. Journal of Marketing, p.12-19, Apr. 1972. 